\title{
Magnetic Resonance Imaging in Myocardial Fibrosis Related to Ischemic Events
}

\author{
Elisabeta Himcinschi', Elena Beganu', Lehel Bordi', Roxana Hodas'1, Theodora Benedek',2 \\ ${ }^{1}$ Center of Advanced Research in Multimodality Cardiac Imaging, Cardio Med Medical Center, Tîrgu Mureș, Romania \\ 2 University of Medicine and Pharmacy, Tîrgu Mureș, Romania
}

\section{CORRESPONDENCE}

\section{Elena Beganu}

Str. 22 Decembrie 1989 nr. 76

540124 Tîrgu Mureș, Romania

Tel: +40 265217333

E-mail: beganu.elena@yahoo.com

\section{ARTICLE HISTORY}

Received: July 14, 2017

Accepted: August 7, 2017
Elisabeta Himcinschi • Str. 22 Decembrie $1989 \mathrm{nr} .76$ 540124 Tîrgu Mureș, Romania. Tel: +40 265217333. E-mail: eli_himcinschi@yahoo.com

Lehel Bordi • Str. 22 Decembrie 1989 nr. 76, 540124 Tîrgu Mureș, Romania. Tel: +40 265217 333. E-mail: bordi_lehel:gmail.com

Roxana Hodas • Str. 22 Decembrie 1989 nr. 76 540124 Tîrgu Mureș, Romania. Tel: +40 265217333. E-mail: roxana.hodas@yahoo.ro

Theodora Benedek • Str. Gheorghe Marinescu nr. 38 540139 Tîrgu Mureș, Romania. Tel: +40 265215551.

E-mail: theodora.benedek@gmail.com

\begin{abstract}
Given the higher amount of detail it offers, the use of magnetic resonance (MR) in the field of cardiology has increased, thus leading to a decrease in the use of invasive and irradiating methods for diagnosing various cardiovascular disorders. The only precautions for MR imaging are metallic implants and advanced-stage chronic kidney disease. For the acquisition of clear and dynamic myocardial images, methods such as spin echo imaging for anatomical description, steady-state free precession imaging for the assessment of ventricular cavity size and function, flow velocity encoding for blood flow measurements, radiofrequency tagging for dynamics, and even spectroscopy for metabolism evaluation are used. Cardiac magnetic resonance (CMR) is considered the gold standard imaging method for the anatomical characterization of the heart and obtaining information related to myocardial dynamics. In case of ischemic events, CMR is used for a detailed description of the necrotic area and the complications, and for tracking the ventricular remodeling. By administrating a contrast agent (gadolinium), the difference between sub-endothelial and transmural infarctions can be distinguished, highlighting even microvascular lesions responsible for the extension of the necrosis. The assessment of the dynamics of ventricular remodeling and viability through late gadolinium enhancement (LGE) technology highlights the area of fibrosis and the occurrence of late complications.
\end{abstract}

Keywords: cardiac magnetic resonance, ischemic events, myocardial viability, ventricular remodeling, fibrosis

\section{INTRODUCTION}

Initial medical usage of magnetic resonance imaging (MRI) was for the imaging of stationary organs within the body (e.g., the brain). However, the latest technical advances, such as sophisticated ECG- and respiratory gating (motion suppression methods) and faster acquisition rates, have facilitated the acquisition of high-quality cross-sectional images of the heart and blood vessels, developing into a new field called cardiovascular magnetic resonance (CMR). ${ }^{1}$

\section{CARDIAC MAGNETIC RESONANCE TECHNIQUES}

Although many techniques are used in MRI studies, CMR is peculiar due to the rapid movements of the cardiac walls and a high-speed blood flow, which 
produces artifacts during image acquisition. On the contrary, real-time CMR methods have a fast acquisition time (the entire image is acquired in $<100 \mathrm{msec}$ ), but are severely limited by spatial and temporal resolution. Widely used methods in this setting include spin echo imaging, steadystate free precession (SSFP) imaging, flow velocity encoding, and radiofrequency (RF) tagging.

Spin echo imaging, also described as "black blood approach" because of the contrast between the bright cardiac walls and the hyposignal of the blood, is used for obtaining an anatomical description, especially in suspicion of fatty infiltration of the right ventricular wall, seen in arrhythmogenic right ventricular cardiomyopathy (ARVC). ${ }^{2}$

SSFP imaging is comprised of images in which the blood is hyperintense, and the cardiac wall is viewed as a hyposignal. This technique has the advantages of presenting high temporal $(<30 \mathrm{msec})$ and spatial $(2 \mathrm{~mm}$ in plane) resolution cine images, obtained during a single breath-hold, but over several cardiac cycles. The method is used for the assessment of ventricular cavity sizes and function, ventricular mass, intracardiac shunts, as well as for the evaluation of valve function and intracardiac masses. ${ }^{1}$

The flow velocity encoding method, also known as "phase contrast", is used for measuring blood flow in pathological settings such as valvular diseases, intracardiac shunts, or arterial stenosis. ${ }^{3}$

$\mathrm{RF}$ tagging is used for the precise assessment of myocardial dynamics. ${ }^{4}$

Other complementary CMR techniques use contrast agents (such as gadolinium) and MRI spectroscopy for evaluating myocardial metabolism. The ECG gating technique optimizes the spatial resolution for a better anatomical description during data acquisition and superposition during several cardiac cycles, and additional respiratory gating enables longer image acquisitions by monitoring the patients' breaths (diaphragm muscle movement or thorax movement). ${ }^{5}$

\section{CLINICAL APPLICATIONS OF CMR}

CMR presents an increased diagnostic accuracy in case of aortic diseases but is limited to hemodynamically stable patients. ${ }^{6}$ Also, MR angiography is of peculiar importance as a noninvasive non-radiating tool for the identification and evaluation of coronary artery anomalies or coronary aneurysms. ${ }^{7}$ On the other hand, CMR is the test of choice for certain pericardial diseases, such as non-calcified constrictive pericarditis, tumor invasion of the pericardium, and congenital absence of the pericardium. However, echocardiography provides comparable diagnostic rates and a more increased availability. ${ }^{8-11}$

CMR is considered the gold standard method for the anatomical characterization of the myocardium and cardiac chambers and the functional assessment of motion wall abnormalities, while the use of gadolinium facilitates the assessment of myocardial scars and fibrosis. ${ }^{12}$

Late gadolinium enhancement (LGE) has been shown to be a good predictor of major acute cardiovascular events (acute or chronic myocardial infarction). LGE (with image acquisition in 10-20 minutes after contrast injection) can be used for a precise description of the infarct size, with minimal inter- and intraobserver variability. A multicenter, double-blinded, randomized trial showed a contrast dosedependent rise of sensitivity and accuracy up to $94 \%$ and $99 \%$ respectively, compared with $11 \%$ in cases where no contrast was used. ${ }^{13}$

A study that compared CMR with LGE to single photon emission computed tomography (SPECT) in patients with coronary artery disease showed a superiority of the former in quantifying the size of the infarction $(92 \%$ vs. $28 \%$ for subendothelial myocardial infarction and similar detection rates for transmural MI). ${ }^{14}$ Another study showed better results for small infarcts (92\% vs. $69 \%)$ and non-anterior infarcts (98\% vs. $84 \%) .{ }^{15}$

Also, subendocardial and transmural LGE is significant in ischemic cardiomyopathy, while isolated mid-wall or epicardial enhancement suggests non-ischemic etiologies. ${ }^{16}$ Electrocardiography and echocardiography for the detection of right ventricular infarction in inferior wall MI were shown to have lower sensitivities compared to CMR. ${ }^{17}$

For prognostic purposes, CMR with LGE detects microvascular obstructions related to the extension of an acute myocardial infarction in the risk assessment of repeated cardiovascular events and impaired left ventricular systolic remodeling. ${ }^{16}$ CMR showed similar findings to positron emission tomography (PET) regarding the assessment of myocardial viability. CMR with LGE has been shown to be an effective tool in identifying fibrotic nonviable ventricular myocardium. ${ }^{18}$ A survival study on 144 patients with documented coronary artery disease, with a median follow-up period of 2.4 years, performed a computer-assisted, semiautomatic CMR with LGE evaluation of the peri-infarct zone (2-3 standard deviations from remote regions) compared to the total infarct zone (core + peri-infarct zone) and found the ratio predictive of post-MI mortality (adjusted hazard ratio of 1.42 per $10 \%$ increase, $p=0.03$ ). The study also showed that the high spatial resolution of CMR may permit a noninvasive evalu- 
ation of the peri-infarct region, where the scarred myocardium is interwoven with isolated bundles of surviving myocytes, which might favor ventricular arrhythmias because of electrical remodeling and newly-formed three-dimensional reentry circuits. ${ }^{19}$ While CMR with LGE identifies focal fibrosis, it remains less useful for diffuse fibrosis. A new technique, equilibrium-contrast CMR, was clinically tested in a small cohort and showed a good correlation to histological fibrosis $(r=0.80)$ but a low intrastudy reproducibility (as low as 1\%). ${ }^{20}$

Myocardial viability is a current issue because of the information it can offer on prognosis, on the risk of certain complications that depend on infarction extension, and the subsequent fibrotic remodeling. Kim et al. questioned whether there is a correlation between delayed contrast enhancement and the age of myocardial infarction, as well as the use of CMR to detect viability and irreversible lesions and evaluate the contractile function of the myocardium. ${ }^{21-24}$ The theory was tested on canine models, and their results revealed that in the first 24 hours from the infarction, hyperenhancement occurs both in the necrosis zone and at the borderline that includes the hibernating myocardium. In terms of chronic infarction, the contrast agent has a strict affinity for the fibrosis area, and the imaging delineation of the hibernated myocardium from the necrosis area is a real challenge. Their study has come to the conclusion that in the acute phase, the contrast area is much larger than the infarct, and therefore it is overestimated; at the same time, it comprises the two areas of interest and even surpasses them, without the possibility of delineation. Therefore, a repeated CMR evaluation at 3 days and at 8 weeks following the acute event shows a net decrease in the necrotic area, with an affinity of the contrast agent only for the fibrotic area. However, the conclusion of the study was that the contrast affinity area only reveals necrosis, explained by the fibrosis shrinking process, except for the first 24 hours, when reperfusion and activation of the hibernating myocardium occurs. ${ }^{21}$

CMR may also play a role in the management of patients with acute chest pain and positive but non-significant coronary obstruction, such as acute myocarditis.

Another utility of CMR is the pharmacological stress using dobutamine. A small study on 51 patients with Chagas disease who underwent CMR with LGE found good correlations between the degree of fibrosis and the clinical stage of the disease. ${ }^{25}$

In patients without a known heart disease who present with ventricular tachycardia or left bundle branch block, the differential diagnosis should include ARVC, and con- firmation may be obtained using noninvasive imaging such as CMR with LGE, which reaches a specificity of $98 \%$ according to the latest Task Force criteria for the diagnosis of ARVC (2010).26 Although according to the 1994 Task Force criteria the method had a $100 \%$ sensitivity, it only had a $50 \%$ specificity for diagnosis, which was explained by substantial interobserver variability due to limited experience; some CMR parameters are considered highly specific (almost $100 \%$ ): right ventricle (RV) dilatation, RV systolic movement impairment, RV late enhancement with gadolinium (suggestive for fibrous-fatty tissue), severe modification of RV (segmental dilatation/aneurysms/regional wall motion abnormalities). ${ }^{26,27}$ Regarding ARVC, CMR with LGE is considered a complementary method besides surface ECG and echocardiography.

CMR might have a role in mapping the atrial walls in atrial fibrillation patients. Long-standing continuous AF promotes remodeling processes that lead to electrical and structural changes in the atrium geometry. CMR with LGE may show morphological changes such as heterogeneous fibrosis distribution and/or hypertrophy..$^{28}$

\section{CMR SAFETY}

Compared to computed tomography studies, native MRI produces three-dimensional images, it has intrinsic high contrast (thus, there is a limited use of contrast agent), no ionizing radiation, no interference with bone tissue or lungs. CMR is limited in case of patients with ferromagnetic metallic implants such as aneurysm clips or pacemakers, claustrophobia, or acutely ill patients because the ECG tracing is distorted by the magnetic field, it has a longer acquisition time, and the gadolinium contrast agent is not recommended in case of patients with moderate to severe kidney disease (with an estimated glomerular filtration rate $<30 \mathrm{~mL} / \mathrm{min}){ }^{1}$

\section{CONCLUSIONS}

The use of MRI in the field of cardiology is highlighted by the fact that it is a noninvasive imaging method that provides detailed images of the anatomy and functional parameters of the heart, and it is a safe method, with few medical contraindications or precautions to be taken into consideration. In case of ischemic events, CMR is used for a detailed description of the necrotic area and the complications, as well as for tracking the ventricular remodeling. The assessment of the dynamics of ventricular remodeling and viability through LGE highlights the area of fibrosis and the occurrence of late complications. 


\section{CONFLICT OF INTEREST}

Nothing to declare.

\section{REFERENCES}

1. Maron MS. Clinical utility of cardiovascular magnetic resonance in hypertrophic cardiomyopathy. Journal of Cardiovascular Magnetic Resonance. 2012;14:13.

2. Menghetti L, Basso C, Nava A, Angelini A, Thiene G. Spin-echo nuclear magnetic resonance for tissue characterisation in arrhythmogenic right ventricular cardiomyopathy. Heart. 1996;76:467-470.

3. Beerbaum P, Körperich $H$, Barth P, Esdorn H, Gieseke J, Meyer H. Noninvasive quantification of left-to-right shunt in pediatric patients: phase-contrast cine magnetic resonance imaging compared with invasive oximetry. Circulation. 2001;103:2476-2482.

4. Sayad DE, Willett DL, Bridges WH, et al. Noninvasive quantitation of left ventricular wall thickening using cine magnetic resonance imaging with myocardial tagging. Am J Cardiol. 1995;76:985-989.

5. Hundley WG, Meshack BM, Willett DL, et al. Comparison of quantitation of left ventricular volume, ejection fraction, and cardiac output in patients with atrial fibrillation by cine magnetic resonance imaging versus invasive measurements. Am J Cardiol. 1996;78:1119-1123.

6. Olin JW, Kaufman JA, Bluemke DA, et al. Atherosclerotic Vascular Disease Conference: Writing Group IV: imaging. Circulation. 2004:109:2626-2633.

7. Spittell PC, Spittell JA Jr, Joyce JW, et al. Clinical features and differential diagnosis of aortic dissection: experience with 236 cases (1980 through 1990). Mayo Clinic Proceedings. 1993;68:642-651.

8. Sechtem U, Tscholakoff D, Higgins CB. MRI of the normal pericardium. AJR Am J Roentgenol. 1986;147:239-244.

9. Breen JF. Imaging of the pericardium. J Thorac Imaging. 2001;16:47-54.

10. Kojima S, Yamada N, Goto Y. Diagnosis of constrictive pericarditis by tagged cine magnetic resonance imaging. N Engl J Med. 1999;341:373374.

11. Masui T, Finck S, Higgins CB. Constrictive pericarditis and restrictive cardiomyopathy: evaluation with MR imaging. Radiology. 1992;182:369373

12. Yu EH, Sloggett CE, Iwanochko RM, Rakowski H, Siu SC. Feasibility and accuracy of left ventricular volumes and ejection fraction determination by fundamental, tissue harmonic, and intravenous contrast imaging in difficult-to-image patients. J Am Soc Echocardiogr. 2000; 13:216-224.

13. Kim RJ, Albert TS, Wible JH, et al. Performance of delayed-enhancement magnetic resonance imaging with gadoversetamide contrast for the detection and assessment of myocardial infarction: an international, multicenter, double-blinded, randomized trial. Circulation. 2008;117:629637.

14. Wagner A, Mahrholdt H, Holly TA, et al. Contrast-enhanced MRI and routine single photon emission computed tomography (SPECT) perfusion imaging for detection of subendocardial myocardial infarcts: an imaging study. Lancet. 2003;361:374-379.
15. Thiele H, Kappl MJ, Conradi S, Niebauer J, Hambrecht R, Schuler G. Reproducibility of chronic and acute infarct size measurement by delayed enhancement-magnetic resonance imaging. J Am Coll Cardiol. 2006;47:1641-1645.

16. Rochitte CE, Lima JA, Bluemke DA, et al. Magnitude and time course of microvascular obstruction and tissue injury after acute myocardia infarction. Circulation. 1998;98:1006-1014.

17. Kumar A, Abdel-Aty $\mathrm{H}$, Kriedemann I, et al. Contrast-enhanced cardiovascular magnetic resonance imaging of right ventricular infarction. J Am Coll Cardiol. 2006;48:1969-1976.

18. Klein C, Nekolla SG, Bengel FM, et al. Assessment of myocardial viability with contrast-enhanced magnetic resonance imaging: comparison with positron emission tomography. Circulation. 2002;105:162-167.

19. Yan AT, Shayne AJ, Brown KA, et al. Characterization of the peri-infarct zone by contrast-enhanced cardiac magnetic resonance imaging is a powerful predictor of post-myocardial infarction mortality. Circulation. 2006;114:32-39

20. Flett AS, Hayward MP, Ashworth MT, et al. Equilibrium contrast cardiovascular magnetic resonance for the measurement of diffuse myocardial fibrosis: preliminary validation in humans. Circulation. 2010;122:138-144.

21. Kim RJ, Fieno DS, Parrish TB, et al. Relationship of MRI delayed contrast enhancement to irreversible injury, infarct age, and contractile function. Circulation. 1999;100:1992-2002.

22. Wu KC, Zerhouni EA, Judd RM, et al. Prognostic significance of microvascular obstruction by magnetic resonance imaging in patients with acute myocardial infarction. Circulation. 1998;97:765-772.

23. Taylor AJ, Al-Saadi N, Abdel-Aty H, Schulz-Menger J, Messroghli DR, Friedrich MG. Detection of acutely impaired microvascular reperfusion after infarct angioplasty with magnetic resonance imaging. Circulation. 2004;109:2080-2085.

24. Tarantini G, Cacciavillani L, Corbetti F, et al. Duration of ischemia is a major determinant of transmurality and severe microvascular obstruction after primary angioplasty: a study performed with contrast-enhanced magnetic resonance. J Am Coll Cardiol. 2005;46:1229-1235.

25. Rochitte CE, Oliveira PF, Andrade JM, et al. Myocardial delayed enhancement by magnetic resonance imaging in patients with Chagas' disease: a marker of disease severity. J Am Coll Cardiol. 2005;46:15531558.

26. Marcus FI, McKenna WJ, Sherrill D, et al. Diagnosis of arrhythmogenic right ventricular cardiomyopathy/dysplasia: proposed modification of the Task Force Criteria. Eur Heart J. 2010;31:806-814.

27. SenChowdhry S, Prasad SK, Syrris P, et al. Cardiovascular magnetic resonance in arrhythmogenic right ventricular cardiomyopathy revisited: comparison with task force criteria and genotype. J Am Coll Cardiol. 2006;48:2132-2140.

28. January CT, Wann LS, Alpert JS, et al. 2014 AHA/ACC/HRS guideline for the management of patients with atrialfibrillation: executive summary: a report of the American College of Cardiology/American Heart Association TaskForce on practice guidelines and the Heart Rhythm Society. Circulation. 2014;130:2071-2104. 